\title{
Mobile Health-monitoring System with Inference, Fall Detection, and Cardiovascular Prediction
}

\author{
Yin-Fu Huang*, Chang-Tai Wang, Yu-Chin Yang, Yu-Hsien Cho, \\ Chen-Ju Lai, Huan-Yu Wu, and Wei-Cheng Chen \\ Department of Computer Science and Information Engineering \\ National Yunlin University of Science and Technology \\ 123 University Road, Section 3, \\ Touliu, Yunlin, Taiwan 640, R.O.C.
}

\begin{abstract}
As the lifetime of human being gets longer, the problems of chronic diseases grow more. In order to make sure the health statuses of patients are not getting worse, they must be health-monitored continuously in a long term. In this paper, a mobile health-monitoring system is built for patients in place of traditional healthcaring manners, which not only gives patients more free spaces, but also can save medical resources, diagnose and predict diseases earlier. In the procedures of health-caring in-house and emergency treatment, a series of vital sensors are combined by integrating sensor network and wireless/mobile network technology to continuously transmit physiological signals of patients to a medical center in a real time, and then doctors can monitor the health statuses of patients exactly, thereby proceeding with diagnosing, recovering, and treatments.
\end{abstract}

Indexing terms/Keywords:health-monitoring, health-caring, wireless sensor, mobile network, inference, fall detection, cardiovascular prediction, data mining

Subject Classification:Health Informatics

Type (Method/Approach):Data Mining

Supporting Agencies:Ministry of Science and Technology of R.O.C.

Language: English

Date of Publication: 23-09-2018

DOI: 10.24297/ijct.v17i2.7626

ISSN: $2277-3061$

Volume: 17 Issue: 02

Journal: International Journal Of Computers \& Technology

Website: https://cirworld.com

This work is licensed under a Creative Commons Attribution 4.0 International License. 


\section{Introduction}

As communication technology has made great progress, mobile communication devices are no longer limited to phone-call and are gradually transformed into the devices of receiving multimedia data such as pictures, music, or even videos. Since information can be obtained and spread more easily, a mobile healthmonitoring system in place of traditional health-caring manners can be realized in the real world by integrating with information, communication, and personalized services.

Some similar studies on mobile health monitoring systems have been proposed in the past years, but they were not well developed. They still require medical staff to continuously monitor sensor data from patients. The health monitoring system proposed by the Loughborough University in the United Kingdom lacks for situational processing and reasoning mechanisms for sensor data [1]. The monitoring system developed by the University of Florence in Italy lacks for effective and feasible long-distance monitoring methods because mobility is not enough [2]. Furthermore, although some more advanced monitoring systems already involved mobile health monitoring and situational reasoning, ontology evolutionary feedback was not employed [3, 4]. Therefore, in this study, we propose a mobile health-monitoring system aiming to eliminate the above restrictions and using a mobile platform to remove manual monitoring on sensor data.

Next, due to the decline in fertility and the increase of average life expectancy, the population structure gradually tends to age. The aging of the population will be a serious matter, so the care of the elderly is very important. Among the care of the elderly, fall events should be paid more attention since they may cause new damage or a major cause of stroke, disability, or even death $[5,6]$. In the past, most fall detection techniques used a sensor to detect a fall event. However, it is necessary for the elderly to wear the sensor, and this makes the monitored elderly uncomfortable. Here, based on the built mobile health-monitoring system, we use a set of video-based devices to detect a fall event, and they do not affect the elderly activity $[7,8,9]$.

Besides, due to the incorrect diet and exercise habits of modern people, the number of patients with cardiovascular diseases increases year by year. Since there are many causes of cardiovascular diseases, sometimes it is difficult for a doctor to judge whether a patient has a heart disease. In recent years, the analysis of patient data is becoming more mature, so we use classification methods on patient data with heart diseases, and build a classification-based cardiovascular disease prediction model [10,11, 12, 13]. By finding out the related attributes with heart diseases, doctors can accurately diagnose whether patients are likely to suffer from heart diseases.

The remainder of this paper is organized as follows. In Section 2, we present the system architecture and briefly describe the mechanisms in the architecture. In Section 3, we introduce how to use mobile communication devices as an intermediary for the system and patients to collect their physiological signals, and a health monitoring and inference mechanism. In Section 4, a video-based fall detection mechanism for the elderly is described. In Section 5, a cardiovascular disease prediction mechanism is proposed. Finally, we make conclusions in Section 6.

\section{System Overview}

In this paper, we propose a mobile health-monitoring system consisting of the infrastructure and a health monitoring and inference mechanism, a video-based fall detection mechanism, and a cardiovascular disease prediction mechanism, as shown in Fig. 1. The mobile health-monitoring system uses mobile communication devices as an intermediary for the system and patients to collect their physiological signals. These physiological signals are measured from wireless sensors worn on patients or located at somewhere in houses and transmitted to mobile communications devices such as smart phones, PDAs, notebook computers, and other devices via Bluetooth networks. Then, these signals are propagated to the health-monitoring system through Internet. The system located in a medical center stores the sensor data in a surveillance repository where doctors can monitor the health statuses of patients exactly and further proceed with diagnosing, recovering, and treatments. Even doctors can make use of a context reasoning engine to infer the health 
statuses of patients. Besides, we also collect monitor data used to detect a fall event for the elderly. When detecting a fall event, the mechanism will issue an alarm message not only to an ambulance station but also to family members or relatives. Then, both sides can promptly make a proper response to deal with the situation. Finally, we also develop a cardiovascular disease prediction mechanism to predict different-level heart diseases, according to the collected data from patients with suspected heart diseases.

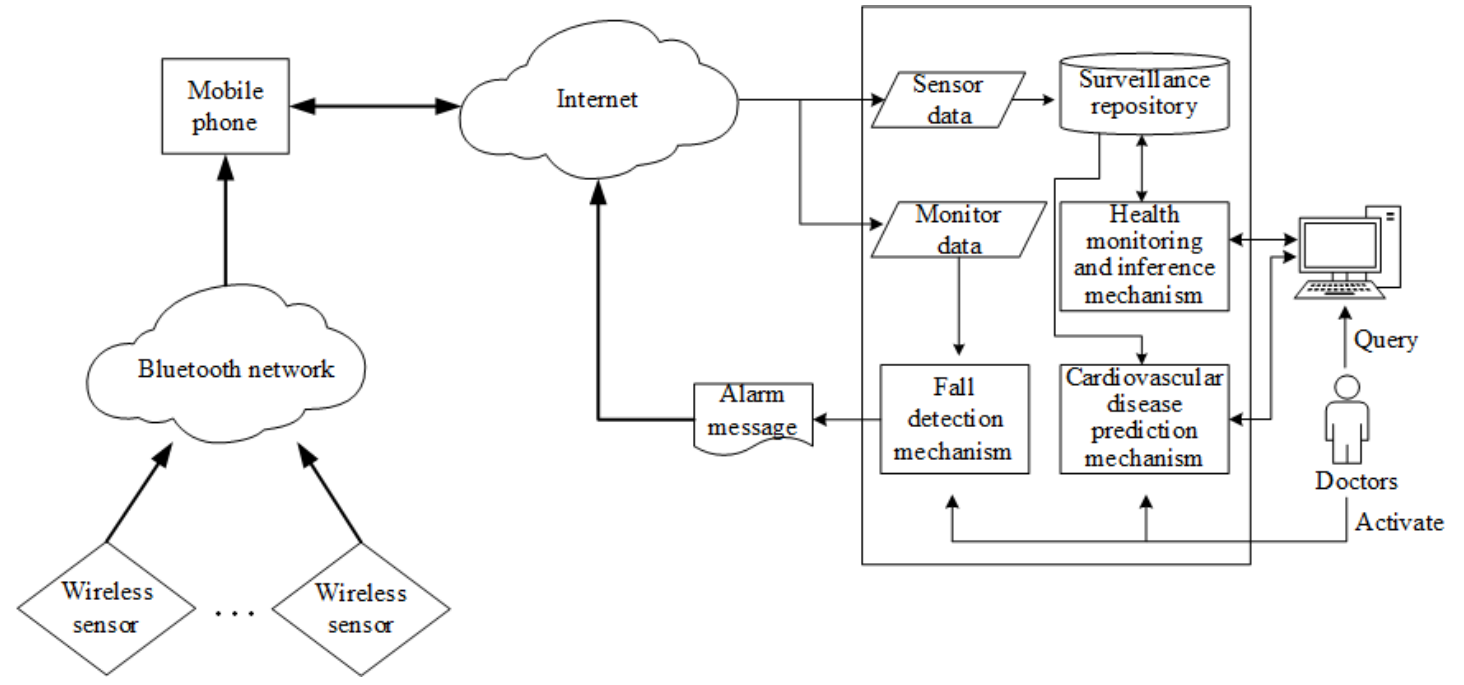

Fig. 1 System architecture

\section{Infrastructure of the Health-monitoring System}

As mentioned, the mobile health-monitoring system uses mobile communication devices as an intermediary for the system and patients to collect their physiological signals. These physiological signals are measured from wireless sensors and transmitted to mobile communications devices via Bluetooth networks. Then, these signals are propagated to the health-monitoring system through Internet. The system located in a medical center stores the sensor data in a surveillance repository where doctors can monitor the health statuses of patients exactly and further proceed with diagnosing, recovering, and treatments. Even doctors can make use of a context reasoning engine and the knowledge base built in the surveillance repository to infer the health statuses of patients, to strengthen patient care, and to promote self-health management and prevention. With the help of the system, not only patients can avoid the recurrence of diseases, but also doctors can learn and understand the symptoms of diseases.

The system accesses the collected data in the repository every pre-set time interval and analyzes them according to the pre-set rules in the knowledge base. Once the data show that the physical conditions of patients are abnormal, the system will issue an alert to inform patients and health-care workers of doing further treatment in order to avoid accidents [14]. The system can be divided into two subsystems: 1) data capture, transmission and user interface and 2) health monitoring and inference mechanism, respectively.

\subsection{Data Capture, Transmission and User Interface}

The goal of data capture and transmission is to extract the measured data of sensors, to convert into an easy-understanding format, and then to transmit to the remote repository for facilitating further analysis and warning. Besides, the health-monitoring user interface as shown in Fig. 2 authenticates the identity of users for privacy and independence and includes five parts in the screen: 1) patient general information such as social ID, name, doctor name, case history ID, and email, 2) detailed case history, 3) sensor type, mobile phone, and physiological data such as systolic pressure, diastolic pressure, and heart rate, 4) alarm level, and finally 5) treatment. 




Fig 2. Health-monitoring user interface

This subsystem extracts the physiological signals of patients, then transmits them to the remote repository, and finally does classification and storage according to different users. In advance, patients have to log in and create personal basic information. When the measured data of sensors are transmitted to a mobile phone via the Bluetooth network, then these signals are propagated to the health-monitoring system through Internet. Finally, doctors can monitor the health statuses of patients exactly and further proceed with diagnosing, recovering, and treatments, as shown in Fig. 3.

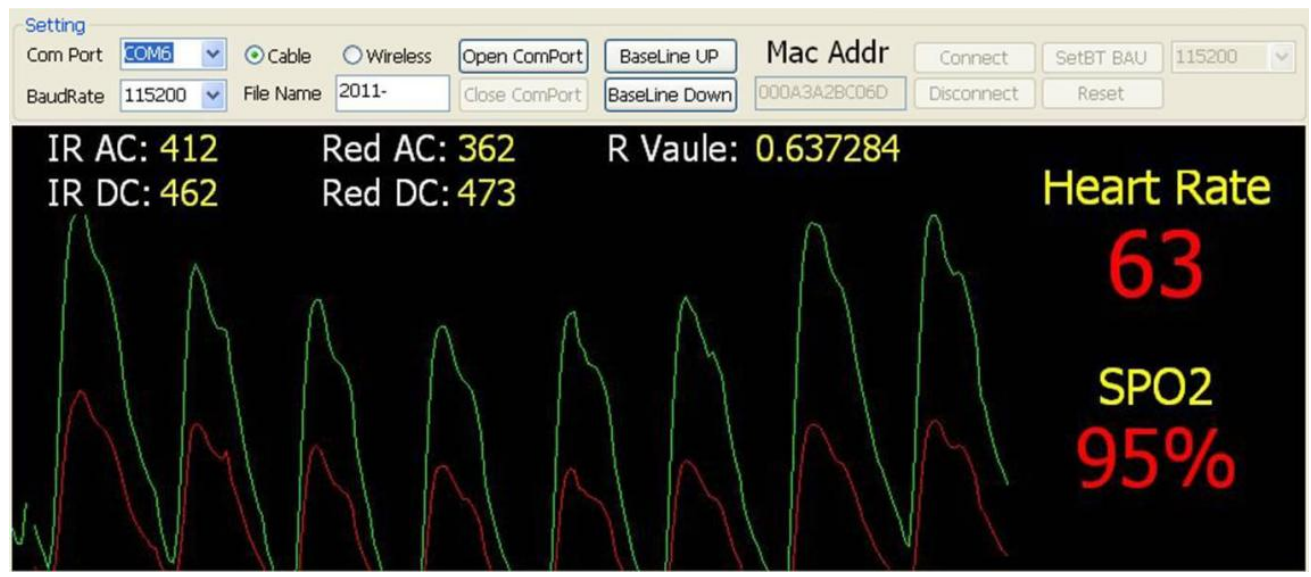

Fig. 3 Health statuses of patients

\subsection{Health Monitoring and Inference Mechanism}

The goal of health monitoring and inference is to store the measured data of sensors in the remote repository and the to infer the health statuses of patients using the rules generated by an AI inferring engine after realizing the high-level semantics of sensor data $[2,15,16,17]$. This subsystem can implemented using 2 modules: 1) database module storing the general information and physiological data of patients, and the analysis results of the inference module, and 2) inference module inferring whether the physical conditions of patients are abnormal according to their physiological data and the pre-set rules in the subsystem.

This subsystem analyzes the latest physiological data of patients every pre-set time interval (e.g., every 30 seconds); if there is no new updated data in the repository, it would access the last measured data. Besides, the analysis rules are also pre-set; e.g., High blood pressure is preset as the measured value higher some threshold. Once abnormal data appear in the analysis, the subsystem immediately issues an alarm message to remind patients and health care workers of if it is necessary to do emergency care and further first aid treatment. 


\section{Video-based Fall Detection Mechanism}

In this section, we propose a video-based fall detection mechanism for the elderly, which applies image processing technology on video images captured from video monitor devices to extract important features and further analyze whether a fall event occurs. Here, Microsoft Kinect sensors are used as video monitor devices to extract important feature points of a human skeleton, and through the calculation of feature points and mobile vector analysis, a fall event can be detected and family members can be notified and reminded of the fall accident through Google cloud messaging services. The mechanism is divided into three parts: 1) feature point extraction module screening out the feature points for a fall event, 2) fall detection module determining different fall situations, and 3) accident notification module transmitting alarm messages to family members and ambulance stations.

\subsection{Feature Point Extraction}

In order to achieve the real-time monitoring of a fall event for the elderly, multiple Microsoft Kinect sensors are deployed at their living spaces in houses. A Kinect sensor records 20 feature points of a human skeleton as shown in Fig. 4, which are used in the subsequent analysis.

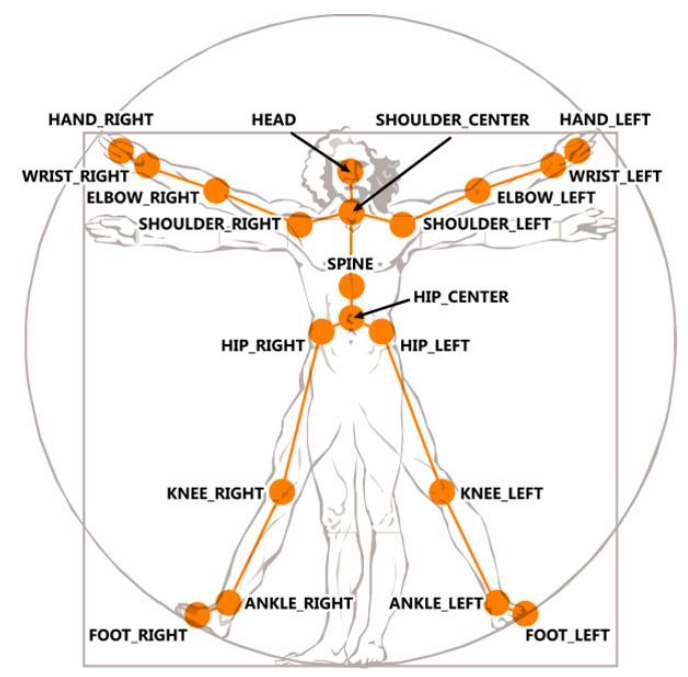

Fig. 420 feature points of a human skeleton

\subsection{Fall Detection}

The feature points obtained from a Kinect sensor are fed into a fall detection module to analyze whether a fall event occurs. When the fall accident occurs, the fall detection mechanism can make a proper response (i.e., transmit alarm messages to notify family members and ambulance stations to deal with the accident). In the fall detection module, five possible fall situations are considered such as 1) backward fall, 2) forward fall, 3) sideward fall, 4) backward fall with laydown, and 5) forward fall with laydown. These detailed fall situations are attached to alarm messages to provide doctors in ambulance stations to do prompt emergency treatment. As mentioned, rather than using a classification method, we directly analyze the feature points obtained from a Kinect sensor to judge a fall event as shown in Fig. 5, so no training set is required in the fall detection module. In the fall event tests, we achieve more than $80 \%$ accuracy in each fall situations. 


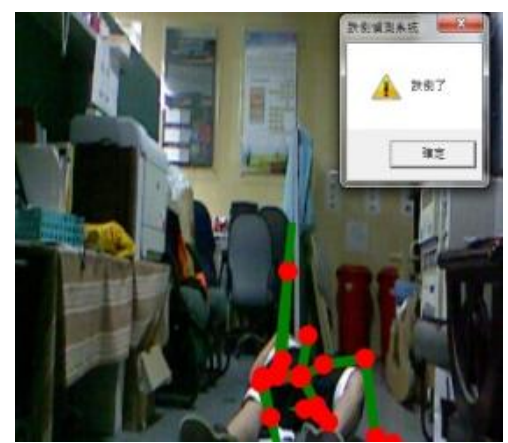

Fig. 5 Fall events

\subsection{Accident Notification}

For the fall accident notification, GCM (Google Cloud Messaging) is used to deliver data (e.g., alarm messages) from servers to Android Apps. The data could be a light-weight message used to inform an Android App to retrieve a new data from the servers; or it could be a message with loaded data (up to $4 \mathrm{~KB}$ ) for instant messaging Apps to receive messages directly. As shown in Fig. 6, the GCM service is divided into five steps: 1) APP is registered with GCM by sending Sender ID to GCM, 2) after the registration is successful, GCM returns Reg. ID for subsequent identification, 3) then APP sends the Reg. ID to our server, 4) if the Server wants to send MSG to App via GCM, it sends (API KEY, Reg. ID, MSG) to GCM instead, and 5) GCM sends MSG to the registered mobile phone.

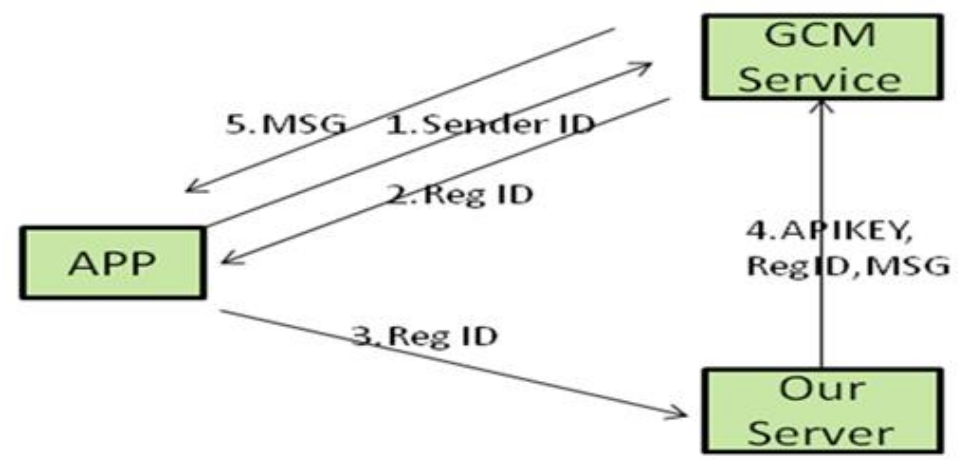

Fig. 6 GCM services

\section{Cardiovascular Disease Prediction Mechanism}

In this section, we use a classification method (i.e., support vector machine) to build a cardiovascular disease prediction mechanism, and predict different-level heart diseases according to the collected data from patients with suspected heart diseases. First, as shown in Fig. 7, we take the heart disease dataset from the UCI Machine Learning Repository as a training set of the classifier [18]. Then, we use a feature selection algorithm called the self-adaptive harmony search algorithm [19] to select relevant features for facilitating heart disease classification. Finally, after training SVM, we conduct a series of experiments and find out the related attributes with heart diseases, and then doctors can accurately diagnose whether patients are likely to suffer from heart diseases. Thus, based on the prediction, doctors can conduct early treatment on these suspected heart disease patients, and reduce the large amount of medical expenses. The user interface of the cardiovascular disease prediction mechanism is shown in Fig. 8. 


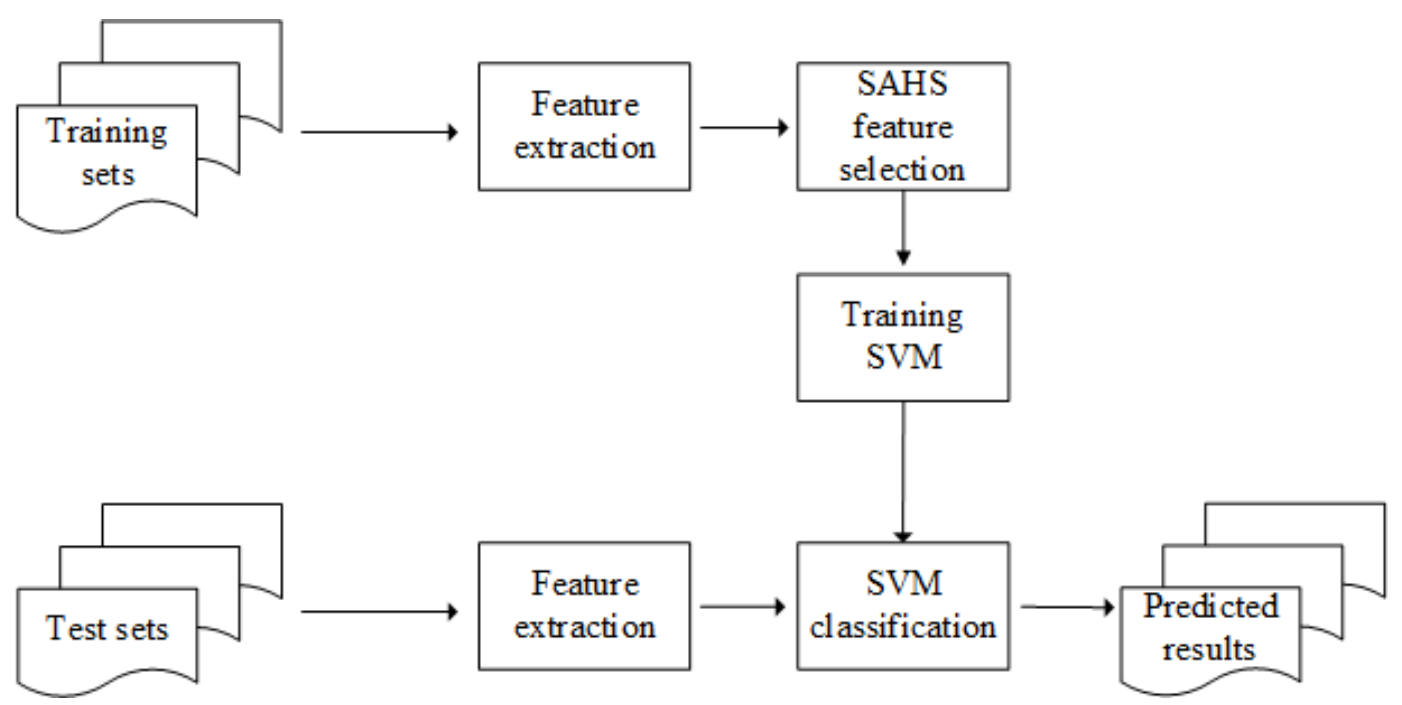

Fig. 7 Architecture of the cardiovascular disease prediction

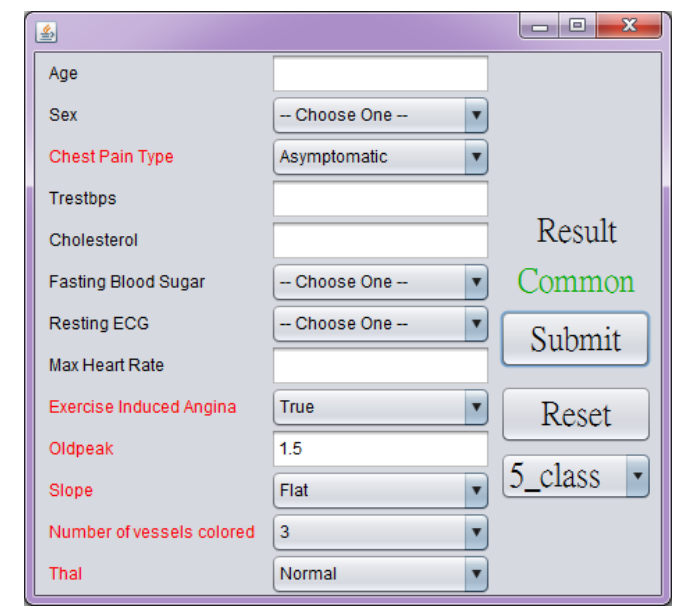

Fig 8. User interface of the cardiovascular disease prediction

\subsection{Heart Disease Dataset}

As mentioned, the heart disease dataset is taken from the UCI Machine Learning Repository as a training set of the classifier. Totally, there are 303 records with 13 attributes, as shown in Table 1.

\subsection{Feature Selection Using the SAHS Algorithm}

In this section, we use a feature selection algorithm called the self-adaptive harmony search algorithm [19] to select relevant features for facilitating heart disease classification. Initially, we have extracted 13 features as shown in Table 1, but not every feature is relevant for the classification [20, 21, 22]. Thus, feature selection is required to improve the classification accuracy. Feature selection algorithms can be categorized into three different approaches: wrappers, filters, and embedded. Here, we use the filter approach [23] to select relevant features since its computational loading is acceptable and the selected classification algorithm could be general. 
Table 1 Attribute descriptions

\begin{tabular}{ll}
\hline \hline \multicolumn{1}{c}{ Attributes } & Descriptions \\
\hline \hline Age & Age of the examiner \\
\hline Sex & Sex of the examiner \\
\hline Chest pain type & Does the examiner feel chest pain? \\
\hline Trestbps (resting blood pressure (in $\mathrm{mm} \mathrm{Hg}$ ) & Blood pressure of the examiner at rest \\
\hline Cholesterol & Cholesterol of the examiner \\
\hline Fasting blood sugar $<120$ & $\begin{array}{l}\text { Before meals (fasting) blood glucose is less } \\
\text { than } 120 \text { mg/dl }\end{array}$ \\
\hline Resting ECG & Static electrocardiogram \\
\hline Max heart rate & Maximum heart rate \\
\hline Exercise induced angina & Does the examiner exercise to cause angina \\
\hline Oldpeak & Degree of ECG from rest to exercise \\
\hline $\begin{array}{l}\text { Slope (the slope of the peak exercise ST } \\
\text { segment) }\end{array}$ & $\begin{array}{l}\text { Electrocardiogram ST in the movement of the } \\
\text { examiner }\end{array}$ \\
\hline Number of vessels colored & $\begin{array}{l}\text { Main color of the blood vessel with X-ray } \\
\text { fluoroscopy } \\
\text { thal }\end{array}$ \\
\hline
\end{tabular}

In our work, the feature selection model consists of two parts: the self-adaptive harmony search (i.e., SAHS) algorithm and the objective function [24], as shown in Fig. 9. Once the original feature set is given, the SAHS algorithm begins to iteratively search a better solution that would be evaluated later by the objective function. Finally, the best solution will be output as the final feature subset.

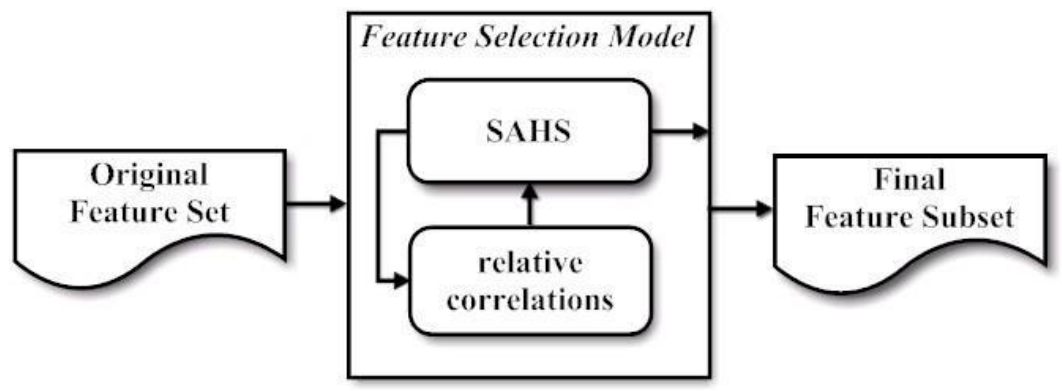

Fig. 9 Feature selection model 
When each new harmony symbolizing a selected feature subset is generated from the SAHS algorithm, the relative correlations are used to evaluate the performance of the selected feature subset. The correlations of the selected feature subset are conducted in two phases; one is the intra-correlation evaluating the mutual correlation between features within the subset, and another is the inter-correlation comparing each feature inside the subset with the corresponding class. If a subset has better performance, it must possess the property of lower intra-correlation and higher inter-correlation. The lower intra-correlation means the features within the subset are relevant, whereas the higher inter-correlation means each feature within the subset is discriminative for the corresponding class.

Here, the well-known measuring formula called mutual information [25] is adopted, which evaluates the degree of the mutual dependence between two variables. The definition for discrete random variables is shown as follows.

$$
I(X ; Y)=\sum_{x \in X} \sum_{y \in Y} p(x, y) \log \left(\frac{p(x, y)}{p_{1}(x) p_{2}(y)}\right)
$$

wherep $(x, y)$ is the joint probability distribution function of $X$ and $Y$, and $p_{1}(x)$ and $p_{2}(y)$ are the marginal probability distribution functions of $X$ and $Y$, respectively. The value of $I(X ; Y)$ is nonnegative; if $I(X ; Y)$ is zero, it means $X$ and $Y$ are independent; otherwise, a high value indicates a strong dependence between $X$ and $Y$.

The intra-correlation within the feature subset is shown as follows.

$$
R I(S)=\frac{1}{C(|S|, 2)} \sum_{i=1}^{|S|} \sum_{j=i+1}^{|S|} I\left(x_{i} ; x_{j}\right)
$$

where $C(|S|, 2)$ is the number of 2-combination on the cardinality of feature subset $S$. The overall correlation within subset $S$ is divided by $C(|S|, 2)$ to present the average correlation between features within subset $S$. The inter-correlation between the feature subset and the corresponding class is shown as follows.

$$
R T(S, y)=\frac{1}{|S|} \sum_{i=1}^{|S|} I\left(x_{i} ; y\right)
$$

where $|S|$ is the cardinality of feature subset $S$, and $y$ is the output class. The overall correlation is divided by the cardinality to derive the average correlation between features and the corresponding class.

Finally, the relative overall correlation combining both the intra-correlation and inter-correlation [22] is shown as follows.

$$
R C(S, y)=\frac{k \times R T(S, y)}{\sqrt{k+k \times(k-1) \times R I(S)}}
$$

wherek is the cardinality of feature subset S. It not only indicates a higher RC value has a better selected feature subset (i.e., it has lower intra-correlation and higher inter-correlation), but also balances the effect of average correlation on different cardinalities of candidate feature subsets. As a result, the RC is chosen as the objective function in the feature selection model.

After using the SAHS algorithm, out of the original 13 features, 5 relevant features are selected for 2 classes of heart diseases and 6 relevant features are selected for 5 classes of heart diseases, as shown in Table 2. These selected features will be used to build the cardiovascular disease prediction model. 
Table 2 Selected features of two cases

\begin{tabular}{clcl}
\hline \hline \multicolumn{1}{c}{ Two classes } & & Five classes \\
\hline \hline 1 & Chest pain type & 1 & Chest pain type \\
\hline 2 & Exercise induced angina & 2 & Exercise induced angina \\
\hline 3 & $\begin{array}{l}\text { Slope (the slope of the peak exercise } \\
\text { ST segment) }\end{array}$ & 3 & $\begin{array}{l}\text { Slope (the slope of the peak exercise } \\
\text { ST segment) }\end{array}$ \\
\hline 4 & Number of vessels colored & 4 & Number of vessels colored \\
\hline 5 & Thal & 5 & Thal \\
\hline & & 6 & Oldpeak \\
\hline
\end{tabular}

\subsection{Classification methods}

Here, LIBSVM software [26] is used to realize the training and test of support vector machine. In the training, two parameters (i.e., penalty parameter $c$ and kernel parametery) should be optimized and determined for support vector machine using grid search and K-fold cross validation method. For the case of 2 classes, penalty parameter $c$ and kernel parameteryare 2048.0 and 0.0001220703125 , respectively. For the case of 5 classes, penalty parameter $c$ and kernel parameteryare 2.0 and 0.0078125 , respectively.

\subsection{Experimental Results}

As mentioned, the heart disease dataset taken from the UCI Machine Learning Repository has 303 records with 13 attributes. For 2 classes of heart diseases, they are negative and positive as shown in Table 3. For 5 classes of heart diseases, they are negative, slight, fair, serious, and very serious as shown in Table 4. Finally, using 10 cross validation, the accuracy rates of these two cases are shown in Table 5.

Table 3 Two classes

\begin{tabular}{lcc}
\hline \hline & Two classes & Number of each class \\
\hline Negative & 164 \\
\hline Positive & 139 \\
\hline
\end{tabular}

Table 4 Five classes

Five classes Number of each class

\begin{tabular}{ll}
\hline Negative & 164
\end{tabular}




\begin{tabular}{lc}
\hline Slight & 55 \\
\hline Fair & 36 \\
\hline Serious & 35 \\
\hline Very serious & 13 \\
\hline
\end{tabular}

Table 5 Accuracy rates of two cases

\begin{tabular}{lcc}
\hline \hline & Two classes & Five classes \\
\hline \hline No feature selection & $84.81 \%$ & $60.39 \%$ \\
\hline Feature selection & $84.48 \%$ & $61.76 \%$ \\
\hline
\end{tabular}

\section{Conclusions}

For elderly people with chronic diseases, long-term monitoring of physiological signals in a home environment is important. Therefore, in this paper, we propose a mobile health-monitoring system consisting of the infrastructure and a health monitoring and inference mechanism, a video-based fall detection mechanism, and a cardiovascular disease prediction mechanism. The system not only gives patients more free spaces, but also saves medical resources, diagnoses and predicts diseases earlier. In this research, we successfully integrate a variety of modules and achieve the purpose of real-time monitoring. Definitely, the system is not only used for long-term care of elderly people with chronic diseases, but also helps the general people to understand their own health and then conducts early treatment. In the future work, we will augment the inference mechanism through the context reasoning engine and the knowledge base to infer the health statuses of patients, to strengthen patient care, and to promote self-health management and prevention

\section{Conflicts of Interest}

We declare that there is no conflict of interest regarding the publication of this paper.

\section{Funding Statement}

This work was supported by Ministry of Science and Technology of R.O.C. under [grant NSC 100-2218-E-224011-MY3].

\section{References}

1. MedGadget, "Another mobile monitoring system promised," 2007, available at http://www.medgadget.com/archives/2007/01/another mobile.html.

2. F. Paganelli and D. Giuli, "An ontology-based context model for home health monitoring and alerting in chronic patient care networks," Proc. the 21st IEEE International Conference on Advanced Information Networking and Applications Workshops, pp. 838-845, 2007.

3. D. Isern, et al., "Home care individual intervention plans in the K4Care platform," Proc. the 21st IEEE International Symposium on Computer-based Medical Systems, pp. 455-457, 2008. 
4. M. Subramanian, et al., "Novel sensor technology integration for outcome-based risk analysis in diabetes," Proc. the 1st International Conference on Health Informatics, Vol. 2, pp. 119-126, 2008.

5. E. Duthie, "Falls," Medical Clinics of North America, Vol. 73, No. 6, pp. 1321-1336, 1989.

6. R. Tideiksaar, Falling in Old Age: Prevention and Management, 2nd ed., Springer-Verlag, 1997.

7. M. Akay, et al., "Unconstrained monitoring of body motion during walking," IEEE Engineering in Medicine and Biology Magazine, Vol. 22, No. 3, pp. 104-109, 2003.

8. B. Krausz and R. Herpers, "Event detection for video surveillance using an expert system," Proc. the 1st ACM Workshop on Analysis and Retrieval of Events/Actions and Workflows in Video Streams, pp. 49-55, 2008.

9. V. D. Shet, et al., "VidMAP: video monitoring of activity with Prolog," Proc. IEEE Conference on Advanced Video and Signal Based Surveillance, pp. 224-229, 2005.

10. A. H. Chen, et al., "HDPS: heart disease prediction system," Proc. Computing in Cardiology, Vol. 38, pp. 557-560, 2011.

11. S. B. Patil and Y. S. Kumaraswamy, "Intelligent and effective heart attack prediction system using data mining and artificial neural network," European Journal of Scientific Research, Vol. 31, No. 4, pp. 642-656, 2009.

12. J. Soni, et al. "Intelligent and effective heart disease prediction system using weighted associative classifiers," International Journal of Computer Science and Engineering, Vol. 3, No. 6, pp. 2385-2392, 2011.

13. G. Subbalakshmi, et al., "Decision support in heart disease prediction system using Naive Bayes," Indian Journal of Computer Science and Engineering, Vol. 2, No. 2, pp. 170-176, 2011.

14. U. Anliker, et al., "AMON: a wearable multiparameter medical monitoring and alert system," IEEE Transactions on Information Technology in Biomedicine, Vol. 8, No. 4, pp. 415-427, 2004.

15. L. W. Goix, et al., "Situation inference for mobile users: a rule based approach," Proc. International Conference on Mobile Data Management, 2007, pp. 299-303, 2007.

16. E. J. Ko, et al., "Ontology-based context modeling and reasoning for U-healthcare," IEICE Transactions on Information and Systems, Vol. E90-d, No. 8, pp. 1262-1270, 2007.

17. Sandia National Laboratories, "Jess, the rule engine for the Java platform," 2013, available at http://www.jessrules.com/.

18. UCI Machine Learning Repository, 2006, available athttp://archive.ics.uci.edu/ml/datasets/Heart+Disease.

19. Y. F. Huang and C. M. Wang, "Self-adaptive harmony search algorithm for optimization," Expert Systems with Applications, Vol. 37, No. 4, pp. 2826-2837, 2010.

20. M. Deriche, "Feature selection using ant colony optimization," Proc. the 6th International Multiconference on Systems, Signals and Devices, pp. 1-4, 2009.

21. R. Diao and Q. Shen, "Two new approaches to feature selection with harmony search," Proc. the IEEE International Conference on Fuzzy Systems, pp. 1-7, 2010. 
22. M. A. Hall and L. A. Smith, "Practical feature subset selection for machine learning," Proc. the 21st Australasian Computer Science Conference, pp. 181-191, 1998.

23. P. L. Lanzi, "Fast feature selection with genetic algorithms: a filter approach," Proc. the IEEE International Conference on Evolutionary Computation, pp. 537-540, 1997.

24. S. Guiasu, Information Theory with Applications, McGraw-Hill, 1977.

25. V. Mitra and C. J. Wang, "A neural network based audio content classification," Proc. the IEEE Region 10 Conference, pp. 1494-1499, 2007.

26. C. C. Chang and C. J. Lin, "LIBSVM: a library for support vector machines," ACM Transactions on Intelligent Systems and Technology, Vol. 2, No. 3, pp. 27:1-27:27, 2011, software available at http://www.csie.ntu.edu.tw/ cjlin/libsvm. 